\title{
Traceable Measurement and Imaging of the Complex Permittivity of a Multiphase Mineral Specimen at Micron Scales using a Microwave Microscope
}

\section{A P Gregory 1 , J F Blackburn 1 , T E Hodgetts 2 , R N Clarke 1 , K Lees ${ }^{1}$, S Plint $^{3}$ and G A Dimitrakis ${ }^{3}$}

\author{
${ }^{1}$ National Physical Laboratory (NPL), Hampton Road, Teddington, Middlesex, TW11 0LW, United \\ Kingdom \\ ${ }^{2}$ Consultant to NPL \\ ${ }^{3}$ Department of Chemical and Environmental Engineering, The University of Nottingham, University Park, \\ Nottingham, NG7 2RD, UK
}

Email: andrew.gregory@npl.co.uk

\begin{abstract}
This paper describes traceable measurements of the dielectric permittivity and loss tangent of a multiphase material (particulate rock set in epoxy) at micron scales using a resonant Near-Field Scanning Microwave Microscope (NSMM) at 1.2 GHz. Calibration and extraction of the permittivity and loss tangent is via an image charge analysis which has been modified by the use of the complex frequency to make it applicable for high loss materials. The results presented are obtained using a spherical probe tip, $0.1 \mathrm{~mm}$ in diameter, and also a conical probe tip with a rounded end $0.01 \mathrm{~mm}$ in diameter, which allows imaging with higher resolution $(\approx 10 \mu \mathrm{m})$. The microscope is calibrated using approach-curve data over a restricted range of gaps (typically between $1 \%$ and $10 \%$ of tip diameter) as this is found to give the best measurement accuracy. For both tips the uncertainty of scanned measurements of permittivity is estimated to be $\pm 10 \%$ (at coverage factor $k=2$ ) for permittivity $\approx 10$. Loss tangent can be resolved to approximately 0.001 . Subject to this limit, the uncertainty of loss tangent measurements is estimated to be $\pm 20 \%$ (at $k=2$ ). The reported measurements inform studies of how microwave energy interacts with multiphase materials containing microwave absorbent phases.
\end{abstract}

Keywords: Scanning microscopes, permittivity measurement, loss tangent measurement, microwave heating, multiphase materials, mineral characterisation, imaging.

\section{INTRODUCTION}

This paper describes imaging of permittivity and loss tangent using a resonator-based Near-Field Scanning Microwave Microscope (NSMM) [1][2]. NSMMs can be used to study multiphase materials, such as composite and functional materials [3][4], biological materials [5] and minerals [6]. In this paper, this is demonstrated by measurements on particulate rock set in an epoxy and polished. The specimen was prepared at the Microwave Process Engineering Research Group at the University of Nottingham, which has strong interests in investigating interactions between microwaves and matter and in developing industrial processes based on microwave heating [7]. The design and test phase of such processes utilises extensive numerical modelling techniques, which are underpinned by the accurate knowledge of the dielectric properties of materials at microwave frequencies. Techniques that are suitable for measurement of permittivity and loss tangent of bulk specimens of rock [8][9][10] have long been available, but NSMMs allow the mineral phases within the rock to be imaged and measured individually. They also make it possible to obtain accurate measurements on mineral phases that can only be found in small volumes. The NSMM described in this paper is based on a microwave resonator that is coupled to a wire probe. Permittivity and loss tangent of a specimen placed at the probe tip are determined from measured shifts in resonant frequency and Q-factor. An alternative type of microwave microscope based on an AFM cantilever has recently been applied to measurements on haematite [6].

The main components of the microscope are shown in Fig. 1. A detailed account of the design and construction of the microscope has recently been published [11]. In the present paper, calibration and imaging measurements are made with a spherical tip $0.1 \varnothing \mathrm{mm}$ manufactured by electro-discharge machining (EDM) [12], and a conical tip manufactured by electrochemical etching (Everbeing T20-100) - see Fig. 2. The conical tip has a rounded end 
with average diameter of $0.01 \varnothing \mathrm{mm}$. Optical microscopy (see Section III(b)) shows that the end of the tip is axially symmetric, but ovoid rather than spherical as a result of the electrochemical etching process. Both probe tips are made from tungsten. Conical tips have a more robust construction than the spherical tip and can be made in smaller sizes to give higher imaging resolution, however, the tip geometry is an important consideration in the model used for calibration and measurement. The shape of tips also has an effect on spatial resolution, which is studied in reference [13].

A mathematical model for the system is required to allow the complex permittivity of measured specimens to be calculated. The resonator must be an integral part of whatever model is used as the relation between complex permittivity and the observed perturbations in frequency and Q-factor must be established. Accurate models that incorporate the tip geometry have been developed for use in Electrostatic Force Microscopy (EFM) experiments for measuring permittivity [14], but these are not directly applicable. It is well established [11] that cavity perturbation theory and an electrostatic model [2] (with modifications that will be summarised in Section III) can be used to obtain accurate measurements of permittivity and loss tangent using spherical tips. Measurements on polar liquids [11] have demonstrated that accurate measurement is possible even for high loss materials. The electrostatic model is developed for an isolated sphere in proximity to a dielectric surface. Computational overheads associated with the electrostatic model are low, so results can be obtained in real time. One of the aims of this paper is to outline a strategy for using this model with the conical tip which gives comparable measurement accuracy to that obtained using the spherical tip. The resonator and probe tip could instead be modelled using the Finite Element Method (FEM), which might in principle be more accurate for the conical tip. However, the large difference in scale between the resonator and the probe tip (for which very fine meshing is needed) limits the accuracy of FEM techniques [15].

Measurements presented in Section VI show that E-fields are not closely confined around probe tips. They are observed to be significant at up to $\approx 1 \mathrm{~mm}$ from the tip. Measurements are sensitive to both local variations of permittivity at a scale smaller than the tip diameter, and also to large-scale dielectric boundaries that are much farther away. This fundamental limitation of a monopole probe tip has important implications for measurements on multiphase materials. Several papers demonstrate that a smaller sensing volume can be obtained using probes based on parallel-plate lines [16][17][18] or miniature bow-tie antennas [19]. These types of probe can be difficult to fabricate to the ideal level of precision, and so problems such as air gaps between the probe tips and specimens, and poor edge definition of metal plating, are likely to occur. To obtain precise measurements of permittivity and loss tangent generally requires implementation of calibration schemes that are tolerant of manufacturing tolerances [17]. Imaging data available in the current literature obtained using such probes is limited.

This paper is structured as follows: In Section II an overview of the design of the microscope is presented. Techniques developed for calibration against dielectric reference materials and extracting the permittivity and loss tangent are summarised in Section III. Measurements by Mineral Liberation Analysis (MLA) that were used to identify the different phases of the rock specimen are described in Section IV, and in Section V NSMM measurements on the rock specimen are presented. The effect of dielectric boundaries is studied in Section VI, and conclusions are presented in Section VII.

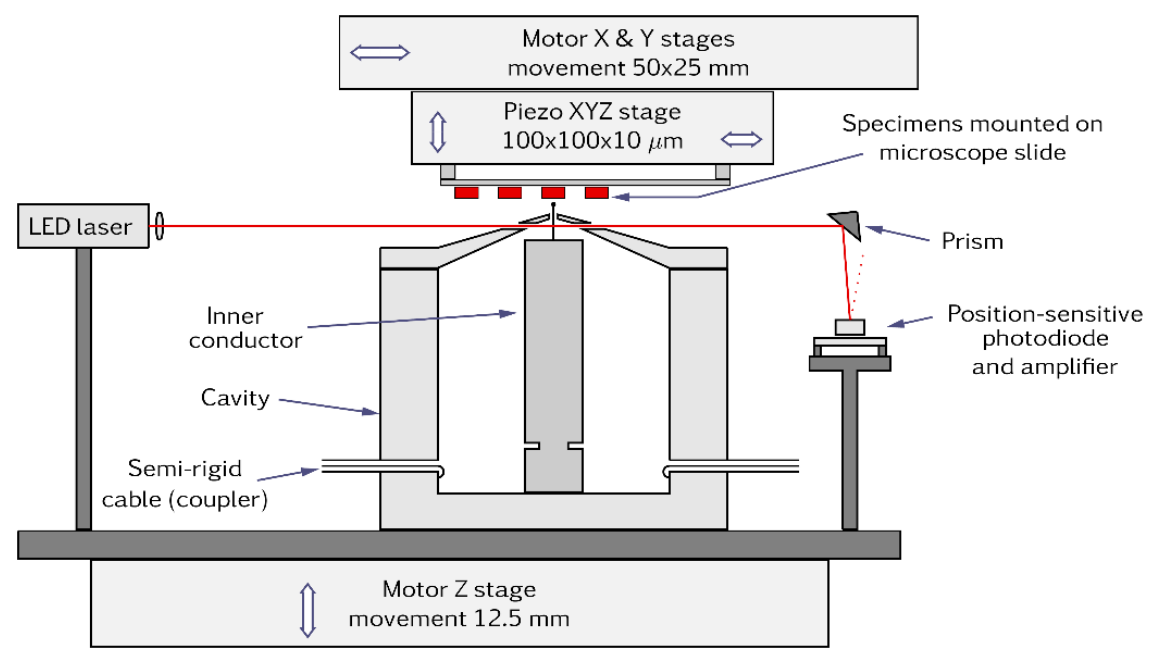

Fig 1: Simplified schematic of the NSMM. 


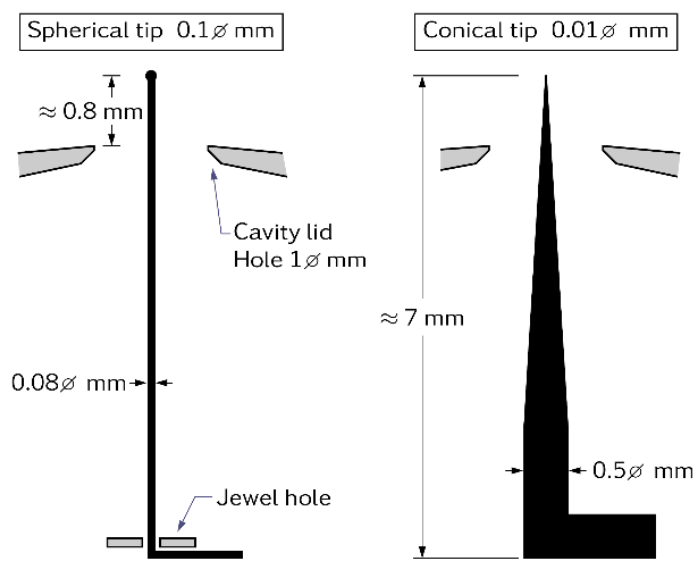

Fig. 2: Tungsten probe tips. These are attached to the inner-conductor using silver paint. For the spherical tip, the assembly is rather delicate. A jewel hole can be used to make the attachment more secure.

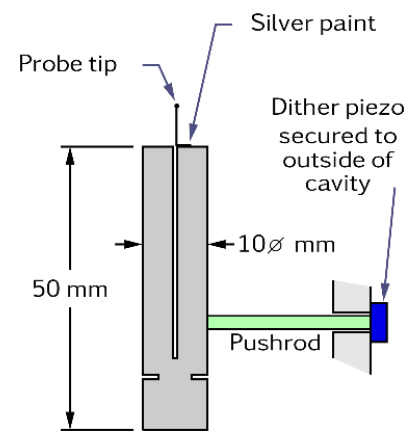

Fig. 3: Inter-changeable inner-conductor assembly

\section{DESIGN OF THE NSMM}

There are a number of published NSMM designs based on quarter-wave coaxial cavities [1][2][20][21], strip-line [22] and dielectric resonators [23]. The instrument described (Fig. 1) uses a quarter-wave coaxial cavity. This has the advantage that a high coupling coefficient between the wire tip and the cavity is easily obtained, so specimens cause comparatively large shifts in the resonant frequency. The cavity is designed to resonate at $\approx 1.2 \mathrm{GHz}$. The Q-factor and resonant frequency of the cavity are obtained via swept-frequency measurements of transmission coefficient $\left(S_{21}\right)$ which are fitted in the complex $S_{21}$-plane by a numerical model - see Fig. 4 . These measurements are made on a Rhode and Schwarz ZVB20 Vector Network Analyser (VNA). The VNA is used uncalibrated but the magnitude of a direct 'thru' correction is used as a normalisation factor to allow unloaded Q-factor to be obtained. Q-factor measurement and the fitting algorithm are described in detail in reference [24]. As the insertion loss of the resonator is high $(\widetilde{S} 30 \mathrm{~dB})$ the VNA can be used at a higher source power setting $(10 \mathrm{dBm})$ than the default to reduce noise while still remaining in its linear range. When measuring high-permittivity specimens at small gaps, the effect of environmental vibrations on measurements is much increased (see Section III) and, in consequence measured $\mathrm{S}_{21}$ Q-circles may have outlying points. The authors' software for fitting resonant frequency and Q-factor allows the largest outliers to be excluded. During calibration, 101 frequency points were used for Q-factor measurement, but this was reduced to 51 during image scans to shorten experiments. Several specimens may be attached to a microscope slide, which is held in place using small magnets to allow it to be removed easily. Motor stages allow scanning over an area of up to $5 \times 5 \mathrm{~mm}$, and also enable switching between specimens to be carried out under computer control. 
Complex permittivity image scans on specimens are made with a minimal gap (estimated to be $\approx 10 \mathrm{~nm}$ ) at which inter-molecular interactions between the tip and the specimen give rise to a shear force which can be detected with sensitive instrumentation. In this paper this is termed 'contact mode'. In the present instrument design, contact mode is detected by means of an optical beam-deflection system based on excitation of a low-frequency (1.3 to $1.4 \mathrm{kHz}$ ) mechanical resonance in the cavity inner-conductor by means of a piezoelectric actuator (referred to as the 'dither piezo'). The inner conductor (Fig. 3) is split by means of a saw cut (width $0.3 \mathrm{~mm}$ ) to give a tuningfork structure that resonates with a Q-factor of approximately 400. Deflections of the shadow of the probe (in laser illumination) are detected with a position-sensitive photodiode and a lock-in amplifier. The probes are secured with silver paint. The optical beam-deflection method enables the length of the wire probe that protrudes beyond the cavity to be short $(<1 \mathrm{~mm})$, which improves sensitivity and reduces error caused by stray field effects. This is a significant advantage compared to an alternative method of obtaining contact mode based on a tuning fork [25]. A measurement of the response of the output voltage of the lock-in amplifier as a function of the Z-axis setting of the piezo stage is shown in Fig. 5. A subsystem based on a feedback circuit drives the Z-axis of the piezo stage so that contact mode can be maintained at the lock point marked in Fig. 5 during scanning. The excitation voltage of the dither piezo can be controlled to ensure that the $S_{21}$ data is not modulated at the dither frequency. In the work presented the excitation voltage is $\leq 100 \mathrm{mV}$. Technical aspects of design of the NSMM are considered in greater detail in reference [11].

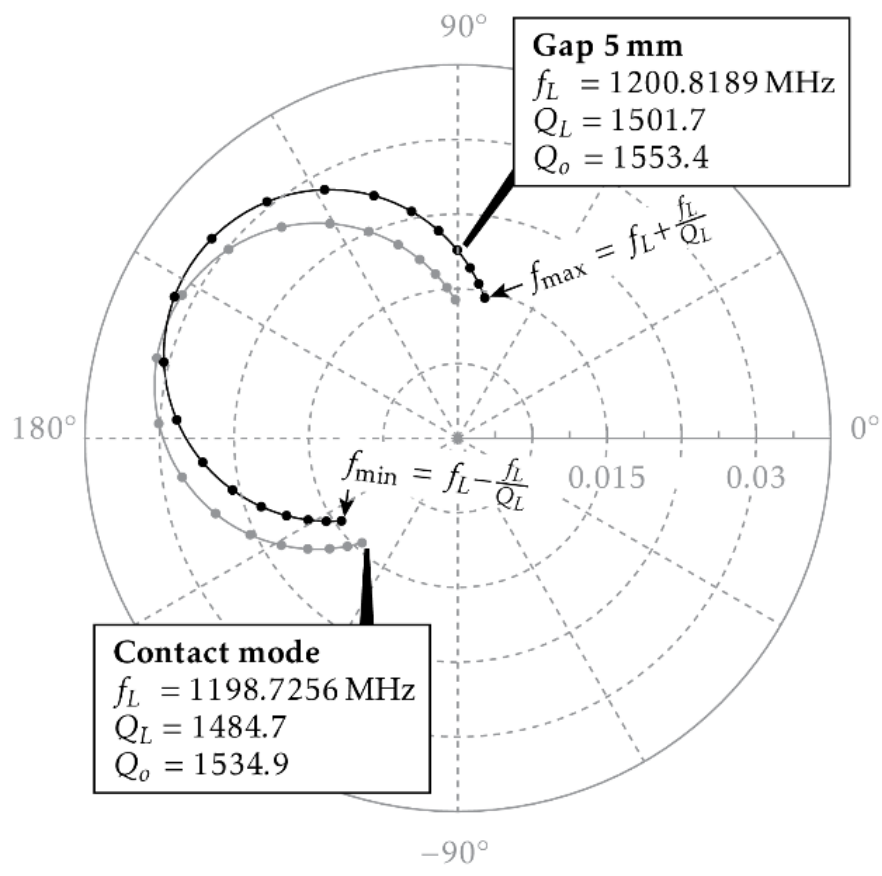

Fig 4: Plots of $S_{21}$ as the frequency is swept across a resonance are circles in the complex plane, referred to as Q-circles [24]. Measurements on a specimen of lanthanum aluminate (LAO) in contact mode and with a 5-mm gap are shown. These sets of data were obtained using the conical tip. The VNA is used uncalibrated but the data shown is normalised to the magnitude of a direct 'thru' connection in place of the resonator. 


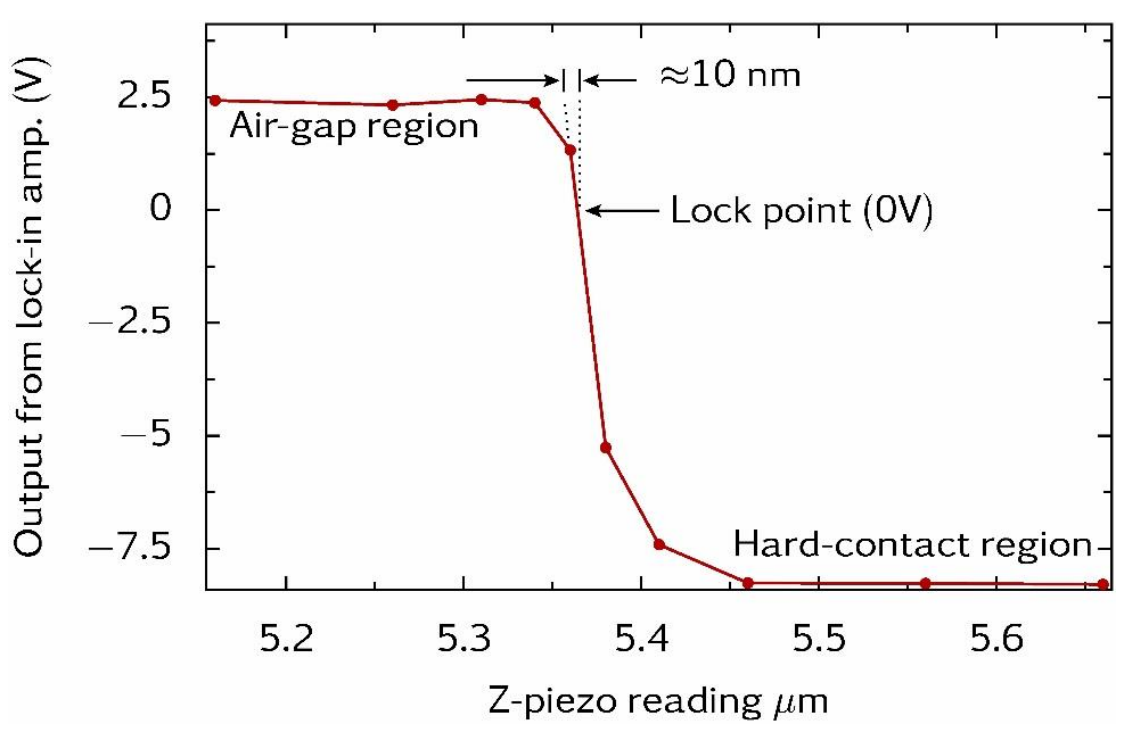

Fig 5: Output voltage from the lock-in amplifier as a function of the Z-axis setting of the piezo stage measured using the conical tip. These measurements were made with the dither-piezo drive voltage set to $25 \mathrm{mV}$.

\section{THE CALIBRATION AND MEASUREMENT PROCEDURE}

A modified version of the model developed by Gao and Xiang (GX) [2] is used for calculating complex permittivity of a specimen-under-test. This is based on an electrostatic image-charge calculation for a spherical tip. A calibration procedure to determine various calibration coefficients is required. This uses measurements of Q-factor and resonant frequency as a function of the separation gap gbetween the probe tip and specimen reference materials - referred to as approach curves. While it is possible to calibrate using only one reference specimen, better accuracy can be obtained using two or three with a range of permittivity values. To obtain an approach curve, the first stage is to establish contact mode and read the position sensor of the $\mathrm{Z}$ piezo. Measurements of the resonant frequency and the Q-factor can then be made at a range of gaps. For the smallest gaps $(g<10 \mu \mathrm{m})$, the movement is generated by the piezo stage in closed-loop mode as this has high positioning accuracy $(5 \mathrm{~nm})$. A motor-driven Z stage enables the resonator and probe tip assembly to be lowered (Fig. 1) to allow larger gaps to be obtained. Calibration measurements at $g=5 \mathrm{~mm}$ (sufficient to ensure that specimens have negligible perturbing effect on the resonance) are recorded as these allow drift in later measurements to be corrected for. The complex permittivity of a specimen-under-test at a single location can be obtained by fitting to approach curve data, or to contact-mode data only. Images are always acquired in contact mode, but even so this is a slow process. It takes the microscope approximately 30 hours to acquire a 200x200 point image, so it is necessary to update the drift correction periodically by re-measuring the frequency and Q-factor at $g=5 \mathrm{~mm}$. The gap between the probe tip and specimens $(\approx 10 \mathrm{~nm})$ is an input parameter for the calibration and measurement process, but in practice has a negligible effect as the probe tips used are comparatively large.

\section{(a) Reference materials}

The NSMM is calibrated [11] from approach curve measurements on isotropic and reproducible materials for which traceable measurements of permittivity are available. High-purity polished fused silica (FSIL), undoped single-crystal yttrium aluminium garnet (YAG) and lanthanum aluminate (LAO) are used in the work described. These materials all have very low loss, which implies that the real part of their permittivity can be taken to be independent of frequency in the range of interest [26]. FSIL and YAG are ideal reference materials, while LAO $\left(\varepsilon^{\prime}=23.9\right)$ is less than ideal but useful because of its comparatively high permittivity. LAO can vary in constitution as rare earth elements are difficult to separate [27]. It is also reported as being slightly anisotropic [27] and to show non-uniformity at a submicron scale due to crystal twinning [28]. High-loss solid materials that are suitable as calibration reference artefacts would have to be homogeneous at the micron scale and have consistent properties, but no such suitable materials have yet been identified. Nevertheless, the loss tangent of high loss materials can be determined by using only low-loss reference materials for calibration and a modified calibration 
model (discussed below). This important finding was an outcome of a study of measurements on polar reference liquids presented in reference [11].

\section{(b) Optical inspection of the probe tips}

One probe tip of each type was examined using an Olympus OLS3100 LEXT confocal microscope system (see Figs. 6 and 7). The spherical tip was observed to be quite accurately shaped (fitted radius $\approx 55 \mu \mathrm{m}$ ) but had surface irregularities (approximately $\pm 2 \mu \mathrm{m}$ ). The end of the conical tip was axially symmetric but not spherical. If only data within $3 \mu \mathrm{m}$ of the end of the tip is considered the fitted radius is $\approx 3 \mu \mathrm{m}$. Using data within $6 \mu \mathrm{m}$ of the end of the tip gives the fitted radius as $\approx 7 \mu \mathrm{m}$.

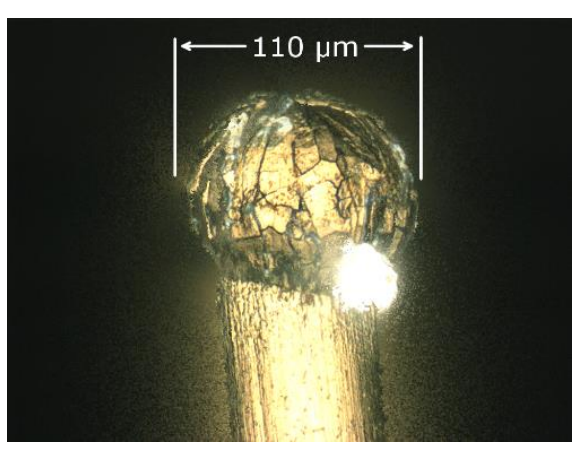

Fig 6: Optical image of the spherical tip.

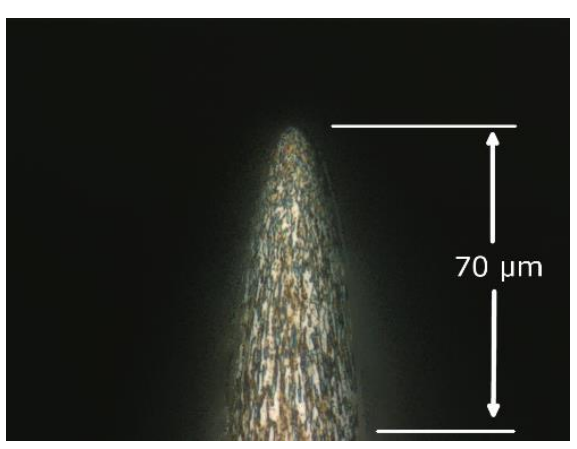

Fig 7: Optical image of the conical tip.

\section{(c) The GXCF model - a modification of the GX model to use complex frequency}

The GX model [2] allows the real part of permittivity to be obtained from the perturbation to the cavity resonant frequency as a function of gap. A relation for calculation of the loss tangent, $\tan \delta$, from changes in $1 / \mathrm{Q}$ is also derived, but this is only applicable for low-loss materials. In reference [11] a 'complex frequency' implementation of the GX model is demonstrated (referred to as the GXCF model). This replaces frequency by 'complex frequency' and allows measurements of complex permittivity $(\varepsilon)$ for high and low loss materials. The complex resonant frequency $\left(f_{r}\right)$ of the cavity is defined by

$$
f_{r}=f_{r}^{\prime}\left(1+\frac{\mathrm{j}}{2 Q}\right)
$$

where $f_{r}{ }^{\prime}$ and $Q$ are the measured (real) resonant frequency and Q-factor. According to the GXCF model, approach curves are described by the equation

$$
f_{r}=f_{\infty}\left(1-A \sum_{n=1}^{\infty} \frac{b t_{n}}{1+\frac{g}{R_{o}}+a_{n}^{\prime}}\right) \text {, }
$$

where $A$ is an amplitude factor, $g$ is the gap between the probe tip and specimen, $R_{o}$ is the tip radius, $b$ is a complex quantity given by $b=(\varepsilon-1) /(\varepsilon+1)$ and $f_{\infty}$ is the complex resonance frequency unperturbed, i.e. extrapolated to $g=\infty$. The quantities $t_{n}$ and $a_{n}^{\prime}$ are calculated by simple formulas - see reference [2] for details. The calibration fits the coefficients $A$ (real or complex), an effective value for the radius $R_{o}$ (real) and $f_{\infty}$ (complex) to the data. In the measurements presented in this paper $A$ is taken to be complex (rather than real) as observations [11] show that improved accuracy for measurements of the loss of low loss materials is obtained. The complex frequency approach enables the loss of specimens to be determined following calibration against LAO, YAG and FSIL, which are low loss materials. This was demonstrated in measurements on BK7 glass $(\tan \delta \approx 0.006)$ and polar liquids $(\tan \delta \approx 1.0)$ that are also reported in reference [11]. 


\section{(d) Calibration data for the spherical and conical tips}

Figs. 8 and 9 show measured approach curves for both tips. In all cases the probe tip is aligned with the centre of the specimens in the XY plane. These graphs also show approach curves calculated from the fitted calibration coefficients using the GXCF model. These use approach curve data for at least two materials over specified gap ranges (points marked by red squares) that are fitted by least squares. The following features can be observed:

(i) Calibrations that are accurate over an extended gap range from contact mode up to 5-mm cannot be established for either probe tip, but much better agreement is obtained for the spherical tip than for the conical tip. Such limitations occur because the GX and GXCF models use a simplified geometry [11]; an electrostatic image charge analysis applied to an isolated perfectly-spherical tip in proximity to a plane dielectric boundary. Experiments have shown that calibrations are more accurate for shorter tips (i.e. ones that do not project far outside the cavity) which suggests that stray field effects are a significant factor. To obtain optimum accuracy for measurement of complex permittivity in

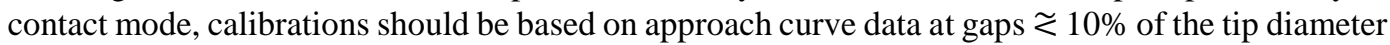
for the spherical tip, and $\approx 5 \%$ for the conical tip.

(ii) Measurements of Q-factor on LAO diverge significantly from the fit at small gaps ( $₹ 1 \%$ of the tip diameter). This can be attributed to sensitivity to mechanical vibrations associated with the optical beam deflection system: when the probe tip is in close proximity to a high permittivity specimen the complex frequency (eqn. (2)) becomes highly sensitive to small changes in the gap, and hence to vibrations. Repeatability measurements (Fig. 10) demonstrate that this is the case. An increase in noise tends to reduce the apparent averaged Q-factor (i.e. a systematic dependence exists) which is attributed to the non-linear dependence of $S_{21}$ on the gap. This interpretation is supported by the observation that if $\mathrm{S}_{21}$ data is modulated by increasing the drive voltage to the dither piezo to $\widetilde{\mathrm{V}} 1 \mathrm{~V}$ the fitted Q-factors reduce if the probe tip is in close proximity $(g \approx 1 \mu \mathrm{m})$ to a high-permittivity material (e.g. LAO). The effects of environmental noise can be minimised by adjustment of the feedback loop settings (e.g. time constants). Improvements brought by such adjustments are most easily discerned when measuring high permittivity materials.

The gap ranges of the approach measurements used for calibration need careful consideration on account of the above observations. The most useful range for calibration is small (e.g. between $1 \%$ and $10 \%$ of the tip diameter). Tables 1 and 2 show measurements of the real part of permittivity $\left(\varepsilon^{\prime}\right)$ and to the loss tangent $(\tan \delta)$ fitted to the calibration data shown in Figs. 8 and 9. In addition, resonant-frequency data for a BK7 glass specimen is presented (it is omitted from the Q-factor graphs Fig. 8(B) and 9(B) for reasons of clarity). An independent measurement using a split-post dielectric resonator (SPDR) [29] on a disc specimen of BK7 at $4 \mathrm{GHz}$ gave $\tan \delta=0.006$. The slightly-lower values of $\tan \delta$ obtained with the NSMM at $1.2 \mathrm{GHz}$ are not inconsistent as some variation with frequency is to be expected. The accuracy of results obtained from contact mode and approach curves are comparable, and the resolution of $\tan \delta$ measurements is observed to be approximately 0.001 .

The effective radius fitted by the calibration using the spherical tip was $70 \mu \mathrm{m}$, which is quite good agreement with the optical measurement $(55 \mu \mathrm{m})$. For the conical tip, the effective radius fitted by the calibration was $14 \mu \mathrm{m}$, which is significantly greater than values estimated optically (3 to $7 \mu \mathrm{m}$ ), but a plausible result given that the GXCF model assumes a spherical geometry. 


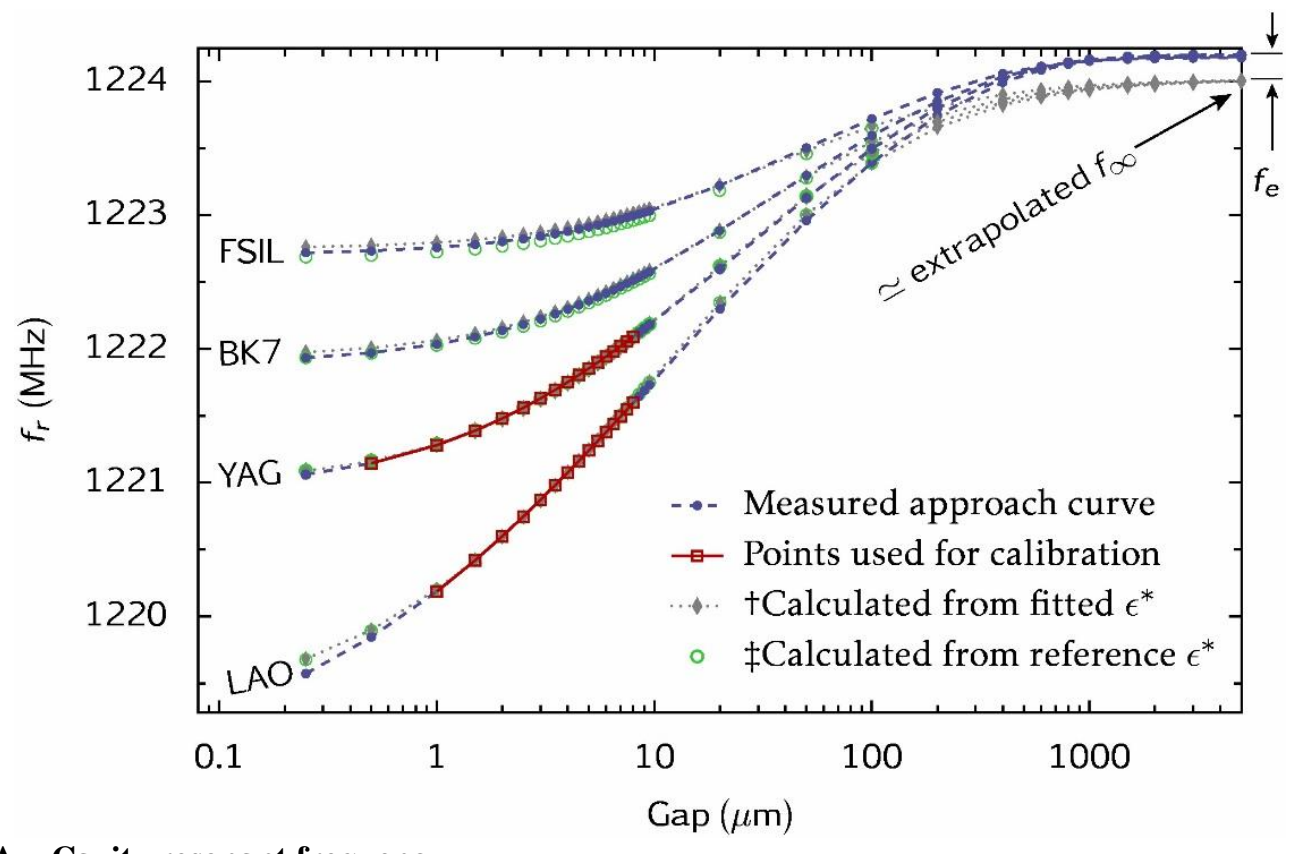

\section{A - Cavity resonant frequency}

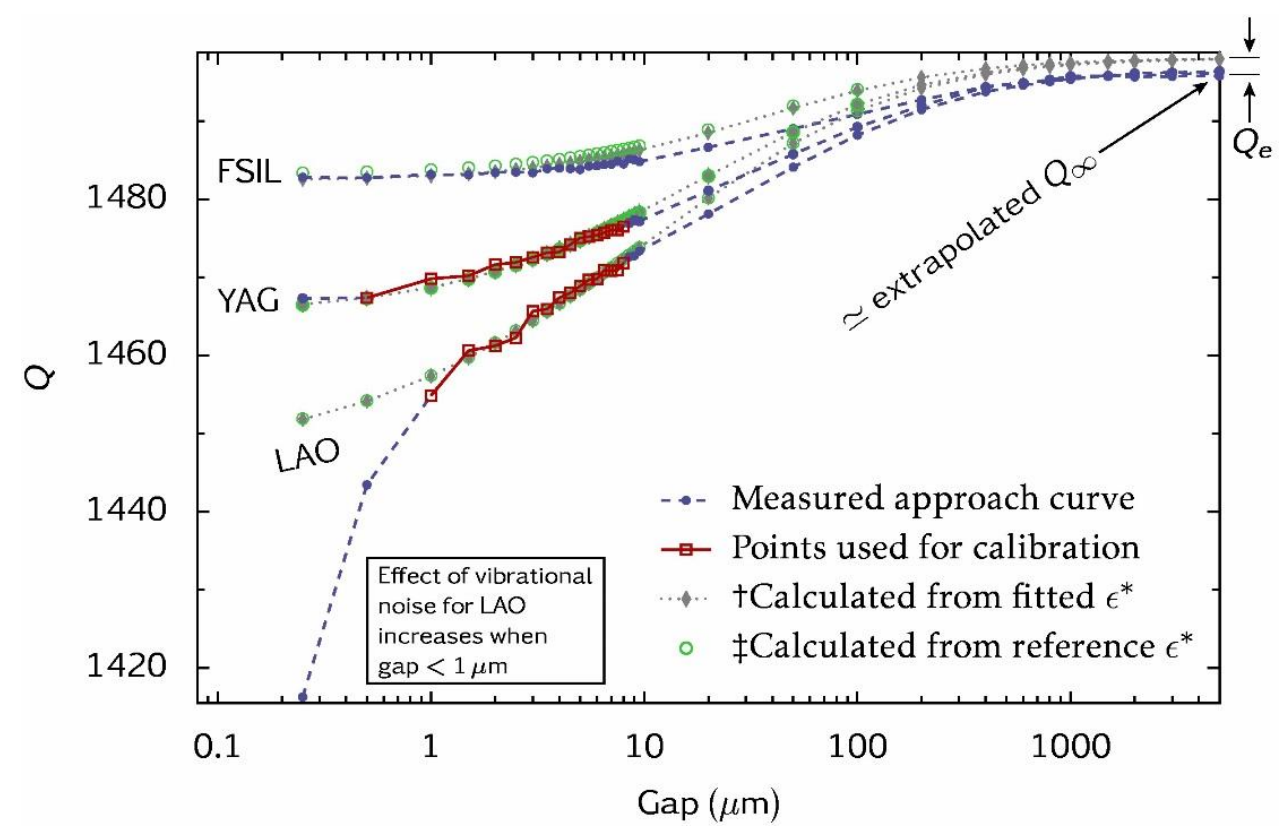

\section{B - Cavity unloaded Q-factor}

Fig 8: Approach curves measurements on the reference specimens obtained with the spherical tip. The microscope was calibrated by fitting the GXCF model to the data points marked in red. The calibration coefficients [11] are $R o(70 \mu \mathrm{m}), f_{\infty}$ $(1224.018+\mathrm{j} 0.408 \mathrm{MHz})$ and $A(0.002194-\mathrm{j} 0.000006)$. The unloaded Q-factors in contact mode for this data set are: 1482.2 (FSIL), 1465.5 (YAG) and 1446.9 (LAO). The quantities $f_{e}$ and $Q_{e}$ are the differences between measured frequency and Qfactor at a 5-mm gap and $f_{\infty}$ and $\mathrm{Q}_{\infty}$ fitted by the calibration.

$\dagger$ Calculated from $\varepsilon^{*}$ fitted to the approach curve (points in range 0.5 to $9.5 \mu \mathrm{m}$ only) using the calibration data. $\$$ Calculated from the reference value of $\varepsilon^{*}$ (Table 1) using the calibration data. For clarity, points at gaps $>200 \mu \mathrm{m}$ are omitted. Q-factor data for the BK7 specimen is omitted as reference data for $\tan \delta$ is only available at $4 \mathrm{GHz}$. 


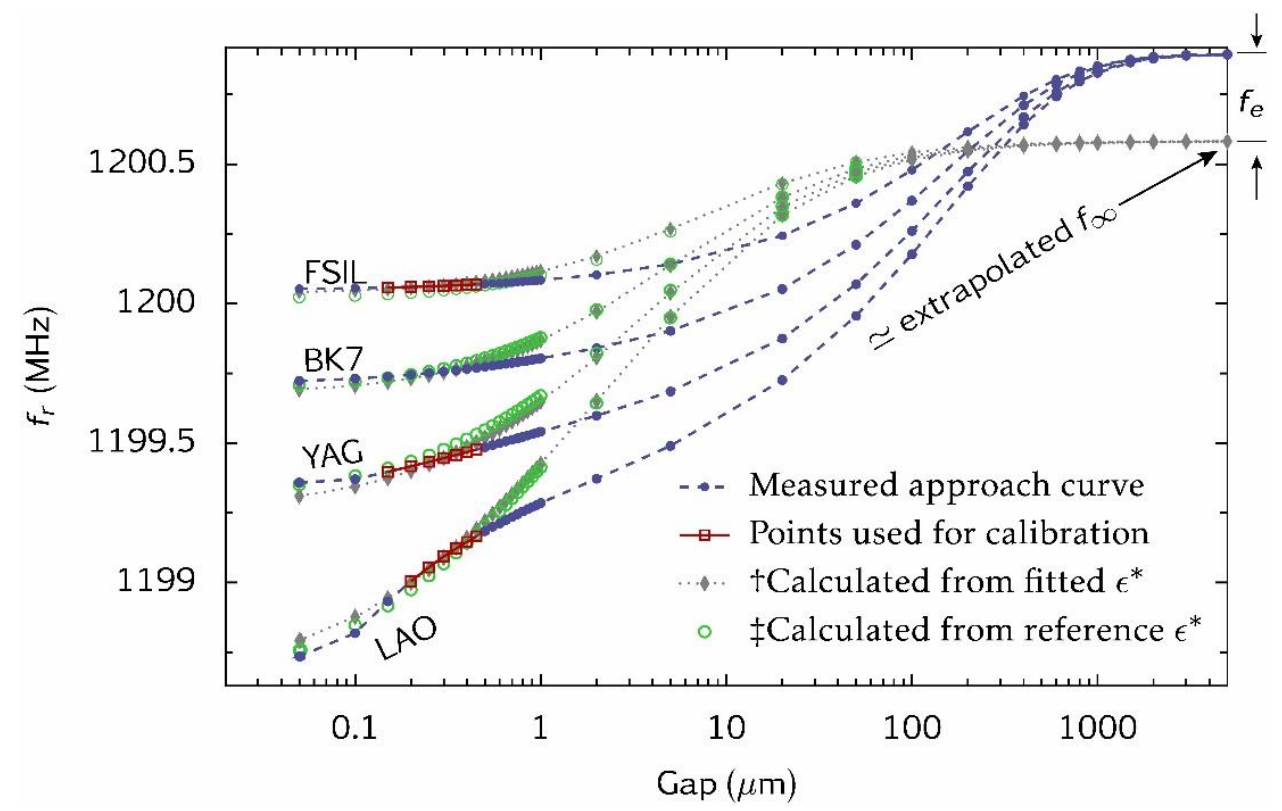

\section{A - Cavity resonant frequency}

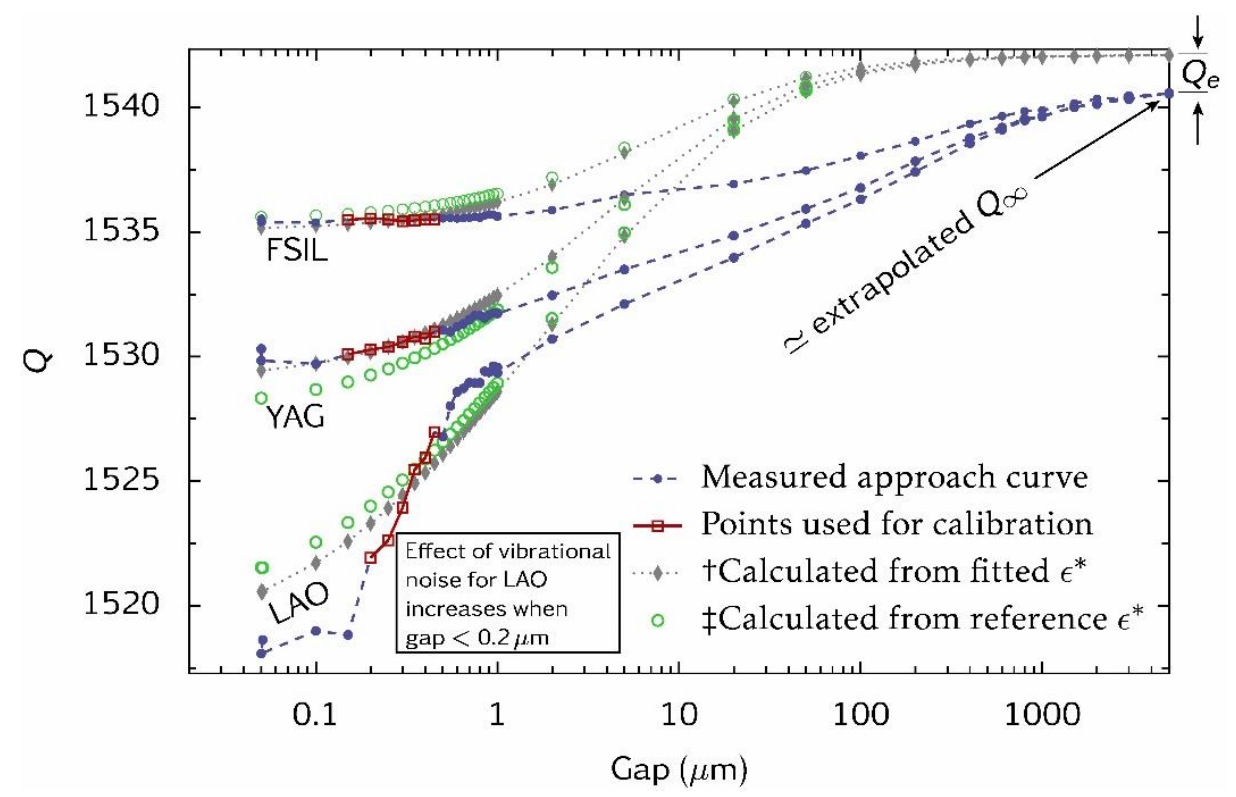

\section{B - Cavity unloaded Q-factor}

Fig 9: Approach curves measurements on the reference specimens obtained with the conical tip. The microscope was calibrated by fitting the GXCF model to the data points marked in red. The calibration coefficients [11] are Ro $(14 \mu \mathrm{m}), f_{\infty}$ $(1200.583+\mathrm{j} 0.389 \mathrm{MHz})$ and $A(0.000941$ - j0.000009). The unloaded Q-factors in contact mode for this data set are: 1535.4 (FSIL), 1530.3 (YAG) and 1522.4 (LAO). The quantities $f_{e}$ and $Q_{e}$ are the differences between measured frequency and Qfactor at a 5-mm gap and $f_{\infty}$ and $\mathrm{Q}_{\infty}$ fitted by the calibration.

$\dagger$ Calculated from $\varepsilon^{*}$ fitted to the approach curve (points in range 0.15 to $0.5 \mu \mathrm{m}$ only) using the calibration data. $\$$ Calculated from the reference value of $\varepsilon^{*}$ (Table 2) using the calibration data. For clarity, points at gaps $>50 \mu \mathrm{m}$ are omitted. Q-factor data for the BK7 specimen is omitted as reference data for $\tan \delta$ is only available at $4 \mathrm{GHz}$. 


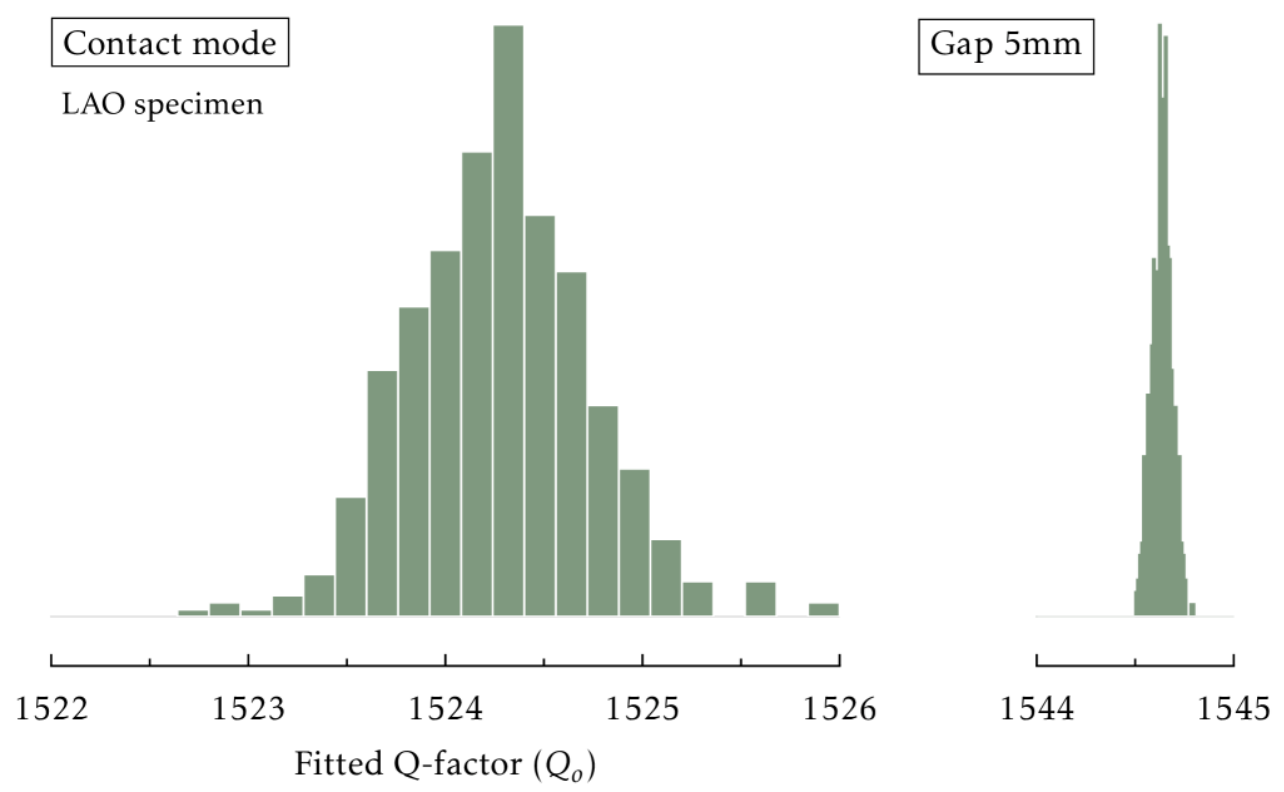

Fig 10: Histograms of 500 Q-factor measurements made with the conical probe tip for the LAO calibration reference specimen in contact mode and at $5 \mathrm{~mm}$ gap. The increase in the width of the distribution of Q-factor measurements at small gaps and in contact mode is largely due to pickup of environmental vibrations by the optical beam deflection system. Sensitivity to vibrations is much increased for high permittivity materials such as LAO. The measurements shown were obtained using a Rhode and Schwarz ZVB VNA with 101 swept frequency points, averaging factor 1, IF bandwidth $100 \mathrm{~Hz}$, source power $10 \mathrm{dBm}$. The insertion loss of the resonator was approximately $31 \mathrm{~dB}$.

Table 1: Measurements on reference materials for the spherical tip from fits to Approach Curves (AP) and Contact Mode $(\mathrm{CM})$ data. The calibration reference specimens were YAG and LAO (gap range $0.5 \mu \mathrm{m}$ to $8.0 \mu \mathrm{m}$ ) and LAO (gap range $1.0 \mu \mathrm{m}$ to $8.0 \mu \mathrm{m}$ ). The AP fits were all made with approach curve data from $0.5 \mu \mathrm{m}$ to $9.5 \mu \mathrm{m}$.

\begin{tabular}{|l|l|l|l|l|l|l|l|}
\hline \multirow{2}{*}{ Spec. } & \multirow{2}{*}{ Nom. $\boldsymbol{\varepsilon}^{\prime}$} & \multicolumn{2}{|c|}{$\boldsymbol{\varepsilon}^{\prime}($ run 1) } & \multicolumn{2}{c|}{$\boldsymbol{\varepsilon}^{\prime}($ run 2) } & \multicolumn{2}{c|}{$\boldsymbol{\varepsilon}^{\prime}($ run 3) } \\
\cline { 3 - 9 } & & AP & CM & AP & CM & AP & CM \\
\hline FSIL & 3.80 & 3.59 & 3.65 & 3.38 & 3.45 & 3.45 & 3.53 \\
\hline BK7 & 6.36 & 6.19 & 6.25 & 5.85 & 5.94 & 5.90 & 6.01 \\
\hline YAG & 10.59 & 10.63 & 10.68 & 10.60 & 10.59 & 10.60 & 10.74 \\
\hline LAO & 23.9 & 24.01 & 24.23 & 24.11 & 24.00 & 24.01 & 24.66 \\
\hline
\end{tabular}

\begin{tabular}{|l|l|l|l|l|l|l|l|}
\hline \multirow{2}{*}{ Spec. } & \multirow{2}{*}{ Nom. $\tan \boldsymbol{\delta}$} & \multicolumn{2}{|c|}{$\tan \boldsymbol{\delta}($ run 1) } & \multicolumn{2}{c|}{$\tan \boldsymbol{\delta}($ run 2) } & \multicolumn{2}{c|}{$\tan \boldsymbol{\delta}($ run 3) } \\
\cline { 3 - 8 } & & AP & CM & AP & CM & AP & CM \\
\hline FSIL & 0.0001 & 0.0005 & 0.0003 & 0.0008 & 0.0005 & 0.0006 & 0.0003 \\
\hline BK7 & $\dagger 0.006$ & 0.0049 & 0.0047 & 0.0046 & 0.0045 & 0.0046 & 0.0045 \\
\hline YAG & 0.00001 & 0.0001 & 0.0001 & 0.0003 & 0.0012 & 0.0000 & 0.0001 \\
\hline LAO & 0.0001 & 0.0005 & 0.0003 & -0.0002 & 0.0008 & 0.0002 & 0.0006 \\
\hline
\end{tabular}

$\dagger$ Reference value obtained at $4 \mathrm{GHz}$. At the frequency of the NSMM measurements $(1.2 \mathrm{GHz}$ ) a change of the order of $\sim 0.001$ is plausible so the NSMM results are consistent. 
Table 2: Measurements on reference materials for the conical tip from fits to Approach Curves (AP) and Contact Mode (CM) data. The YAG and LAO were used for calibration (gap range $0.15 \mu \mathrm{m}$ to $0.5 \mu \mathrm{m}$ ) and LAO (gap range $0.2 \mu \mathrm{m}$ to $0.5 \mu \mathrm{m}$ ). The AP fits were all made with approach curve data from $0.15 \mu \mathrm{m}$ to $0.5 \mu \mathrm{m}$.

\begin{tabular}{|l|l|l|l|l|l|l|l|}
\hline \multirow{2}{*}{ Spec. } & \multirow{2}{*}{ Nom. $\boldsymbol{\varepsilon}^{\prime}$} & \multicolumn{2}{|c|}{$\boldsymbol{\varepsilon}^{\prime}($ run 1) } & \multicolumn{3}{c|}{$\boldsymbol{\varepsilon}^{\prime}($ run 2) } & \multicolumn{2}{c|}{$\boldsymbol{\varepsilon}^{\prime}$ (run 3) } \\
\cline { 3 - 9 } & & AP & CM & AP & CM & AP & CM \\
\hline FSIL & 3.80 & 3.70 & 3.61 & 3.66 & 3.58 & 3.62 & 3.52 \\
\hline BK7 & 6.36 & 6.51 & 6.23 & 6.53 & 6.19 & 6.53 & 6.24 \\
\hline YAG & 10.59 & 11.12 & 10.33 & 11.30 & 10.31 & 11.48 & 10.68 \\
\hline LAO & 23.9 & 23.10 & 22.39 & 22.90 & 21.89 & 22.00 & 21.00 \\
\hline
\end{tabular}

\begin{tabular}{|l|l|l|l|l|l|l|l|}
\hline \multirow{2}{*}{ Spec. } & \multirow{2}{*}{ Nom. $\tan \boldsymbol{\delta}$} & \multicolumn{2}{|c|}{$\tan \boldsymbol{\delta}($ run 1) } & \multicolumn{2}{c|}{$\tan \boldsymbol{\delta}($ run 2) } & \multicolumn{2}{c|}{$\tan \boldsymbol{\delta}(\operatorname{run} 3)$} \\
\cline { 3 - 8 } & & AP & CM & AP & CM & AP & CM \\
\hline FSIL & 0.0001 & 0.0002 & 0.0001 & 0.0005 & 0.0004 & 0.0001 & 0.0001 \\
\hline BK7 & $\dagger 0.006$ & 0.0040 & 0.0039 & 0.0044 & 0.0043 & 0.0043 & 0.0043 \\
\hline YAG & 0.00001 & -0.0001 & -0.0001 & -0.0005 & -0.0006 & 0.0001 & 0.0001 \\
\hline LAO & 0.0001 & 0.0006 & 0.0014 & 0.0002 & -0.0002 & 0.0003 & 0.0004 \\
\hline
\end{tabular}

$\uparrow$ Reference value obtained at $4 \mathrm{GHz}$. At the frequency of the NSMM measurements $(1.2 \mathrm{GHz})$ a change of the order of $\sim 0.001$ is plausible so the NSMM results are consistent.

\section{CHARACTERISATION OF THE ROCK SPECIMEN USING A MINERAL LIBERATION ANALYSER}

A Mineral Liberation Analyser (MLA) was used to characterise the rock specimen [30][31][32][33] so that the phases that were present could be identified prior to measurement with the microwave microscope. MLA is an automated SEM technique which uses the back-scattered electron (BSE) and X-ray signals. The hardware consists of a large chamber SEM with standard peripheral detectors. The MLA at Nottingham comprises an FEI Quanta 600 SEM with an EDAX detector using Genesis 4000 software. The MLA software interfaces with the relevant microscope control software for automated measurement and processing of sequential frames. The MLA measurements are undertaken on polished cross sections of epoxy mounted ore particles. These are generally carbon coated, but low resolution measurements can be made on uncoated surfaces in low vacuum mode.

The first step in measurement is BSE image capture. The BSE image contrast is a function of mean atomic number, allowing 'on-line' background (epoxy) subtraction, and segmentation of mineral areas into areas of differing brightness. In the simplest type of measurement, an X-ray spectrum is collected from each of the greyscaleresolved mineral segments. In complex composites, including minerals of similar greyscale, a grid of X-ray spectra are collected over a certain greyscale range to enhance the ultimate mineral segmentation. The measurement file includes background subtracted, segmented image files for each frame, and one or more X-ray spectra associated with each mineral segment. Off-line classification software pattern-matches the measurement spectra with a pre-determined library of mineral spectra to produce colour classified images for each frame. By analysis of the images, parameters such as mineral percentage, mineral grain size distribution and mineral liberation can be deduced.

The rock specimen used in this study contains a number of different mineral phases set in an epoxy matrix (dimensions 10x10x1 mm). The phases were identified using an MLA. They include apatite, baddeleyite, calcite, chalcopyrite, dolomite, magnetite, olivine and serpentine. Chalcopyrite $\left(\mathrm{CuFeS}_{2}\right)$ and magnetite $\left(\mathrm{Fe}_{3} \mathrm{O}_{4}\right)$ are copper-iron and iron minerals that are mined for metal extraction. Baddeleyite is an uncommon zirconia $\left(\mathrm{ZrO}_{2}\right)$ mineral with monoclinical crystal structure. The remaining phases are common rock-forming minerals. Several $1 \times 1 \mathrm{~mm}$ areas of the specimen that contain examples of the different mineral phases of reasonable size $(\widetilde{>} 0.2 \mathrm{~mm})$ were identified as being appropriate for measurement using the NSMM. One of these is shown in Fig. 11. 


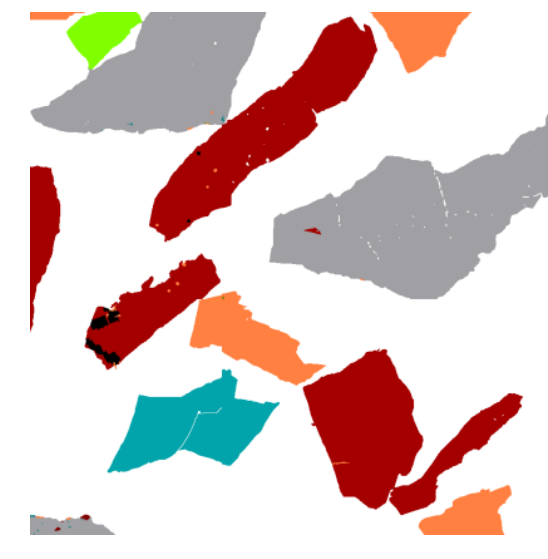

Fig. 11: Mineral phases in a 1x1 mm area.

\section{V. $\quad$ NSMM MEASUREMENTS ON MinERAL PHASES}

Naturally occurring minerals show great variation in both morphology and impurity content. Serpentine is actually a group of minerals that have widely-varying constituents. Sources of dielectric data in the literature for minerals are quite limited, and no data could be found for baddeleyite, olivine and serpentine. For minerals for which literature data is available, a wide range of values for permittivity and loss tangent are reported. The data presented in this paper may be useful as a broad indication of the dielectric properties of the types of mineral that have been measured.

An initial large-area scan (5x5 mm) was made to enable features on the specimen to be matched with the MLA results. The largest mineral phases of each type were identified and measured in $1 \times 1 \mathrm{~mm}(200 \times 200$ points) contactmode scans. Scans over one of these areas using both probe tips are shown in Fig. 12. The drive voltage for the dither piezo was $25 \mathrm{mV}$ (conical tip) or $100 \mathrm{mV}$ (spherical tip) - in neither case sufficient to cause significant modulation of $S_{21}$ measurements. The scales of the plots have been chosen to show a chalcopyrite phase, which is of particular interest as it has much greater loss than any of the other phases. The observed ranges of permittivity and loss tangent, and literature data, are shown in Table 3. For the magnetite phases, the Q-circle is observed to be acutely sensitive to vibrations. This is a clear indicator that magnetite is a conductor; the same observation was made when a metal plate was measured. Repeatable measurements of resonant frequency and Q-factor were not obtainable for magnetite, so permittivity and loss tangent data is not presented. All other phases are assumed to be non-magnetic insulators for which the GX/GXCF theory is devised. It may however be noted that chalcopyrite is reported to be a partial conductor [34].

Fig. 13 shows linear plots in the Y direction to show the $\varepsilon^{\prime}$ and $\tan \delta$ of the chalcopyrite phase. Data is shown for both tips at approximately the same location. The data is extracted from the same data sets used to create the images shown in Fig. 12. It can be observed that the conical tip shows an improved level of detail. Measurement noise is however increased as smaller changes in resonant frequency and Q-factor are measured.

It is interesting to note that the data for the conical probe has spikes at the boundary between the chalcopyrite phase and the surrounding epoxy matrix. This effect was replicated in experiments in which the probe was moved under manual control: locations along the boundary were found at which stable readings of Q-factor as low as 100 could be identified. The evidence shows that this is not a spurious instrumentational effect, but that these are regions of increased loss that the conical tip is able to resolve better than the spherical tip. The reason why $\tan \delta$ is anomalously high at many points on the boundary is not known. No such phenomenon was observed for any of the other mineral phases. Fig. 14 shows a scan across a baddeleyite phase. Table 3 presents a summary of observed $\varepsilon^{\prime}$ and $\tan \delta$ data for selected phases.

A sample of the epoxy matrix material (epofix from Struers, UK) was prepared for measurement using a splitpost dielectric resonator [29] at $4 \mathrm{GHz}$. This gave $\varepsilon^{\prime}=2.94 \pm 0.03$ and $\tan \delta=0.031 \pm 0.001$ (uncertainties given at coverage factor $k=2$ ). 
Table 3: Dielectric measurements on the mineral phases in the rock specimen showing variations. These measurements are made in contact mode at $1.2 \mathrm{GHz}$. One example of each mineral phase is measured (those chosen are among the largest particles, typically 0.3 to $0.5 \mathrm{~mm}$ across). These results are obtained from measurements on several areas of the specimen; not only the $1 \times 1 \mathrm{~mm}$ area shown in Figs. 11 and 12 .

\begin{tabular}{|c|c|c|c|c|c|c|}
\hline \multirow[t]{2}{*}{ Phase } & \multirow[t]{2}{*}{ Tip } & \multirow[t]{2}{*}{ Observed $\varepsilon^{\prime}$} & \multirow[t]{2}{*}{ Observed $\tan \delta$} & \multicolumn{3}{|c|}{ Literature values } \\
\hline & & & & $\varepsilon^{\prime}$ & $\tan \delta$ & Freq. \& Ref. \\
\hline \multirow[t]{2}{*}{ Apatite } & Spherical & $6.6-7.7$ & $0.013-0.016$ & \multirow[t]{2}{*}{9.43} & \multirow[t]{2}{*}{ "0.81 } & \multirow[t]{2}{*}{$1 \mathrm{GHz}[35]$} \\
\hline & Conical & $7.3-7.6$ & $0.014-0.016$ & & & \\
\hline \multirow[t]{2}{*}{ Baddeleyite } & Spherical & $10.5-11.5$ & $+\overbrace{}^{\dagger} 0.015-0.02$ & \multirow[t]{2}{*}{$\neq 24$} & & \multirow[t]{2}{*}{$1 \mathrm{MHz}[36]$} \\
\hline & Conical & $10.5-11.5$ & $\dagger 0.015-0.025$ & & & \\
\hline \multirow[t]{2}{*}{ Calcite } & Spherical & $7-7.5$ & $0.024-0.027$ & \multirow[t]{2}{*}{8.91} & \multirow[t]{2}{*}{0.47} & \multirow[t]{2}{*}{$1 \mathrm{GHz}[35]$} \\
\hline & Conical & $6.4-7.7$ & $0.021-0.023$ & & & \\
\hline \multirow[t]{2}{*}{ Chalcopyrite } & Spherical & $15-16$ & $0.5-0.6$ & \multirow{2}{*}{$\begin{array}{l}10.38 \\
12.2 \\
\end{array}$} & \multirow{2}{*}{$\begin{array}{l}0.223 \\
0.8\end{array}$} & \multirow{2}{*}{$\begin{array}{l}1 \mathrm{GHz}[34] \\
1.2 \mathrm{GHz}[9]\end{array}$} \\
\hline & Conical & $12-13$ & $0.5-0.7$ & & & \\
\hline \multirow[t]{2}{*}{ Dolomite } & Spherical & $6.0-6.2$ & $0.017-0.018$ & \multirow[t]{2}{*}{7.41} & \multirow[t]{2}{*}{2.42} & \multirow[t]{2}{*}{$1 \mathrm{GHz}[35]$} \\
\hline & Conical & $5.9-6.6$ & $0.016-0.020$ & & & \\
\hline \multirow[t]{2}{*}{ Olivine } & Spherical & $6.8-7.0$ & $0.015-0.017$ & & & \\
\hline & Conical & $7.3-7.4$ & $0.017-0.019$ & & & \\
\hline \multirow[t]{2}{*}{ Serpentine } & Spherical & $6.3-6.5$ & $0.03-0.05$ & & & \\
\hline & Conical & $6.0-6.7$ & $0.022-0.024$ & & & \\
\hline
\end{tabular}

$\dagger$ The large peak in $\tan \delta$ shown in Fig 14 is ignored. This is believed to be a vein of another mineral.

$\neq$ For pure monoclinic zirconia. 

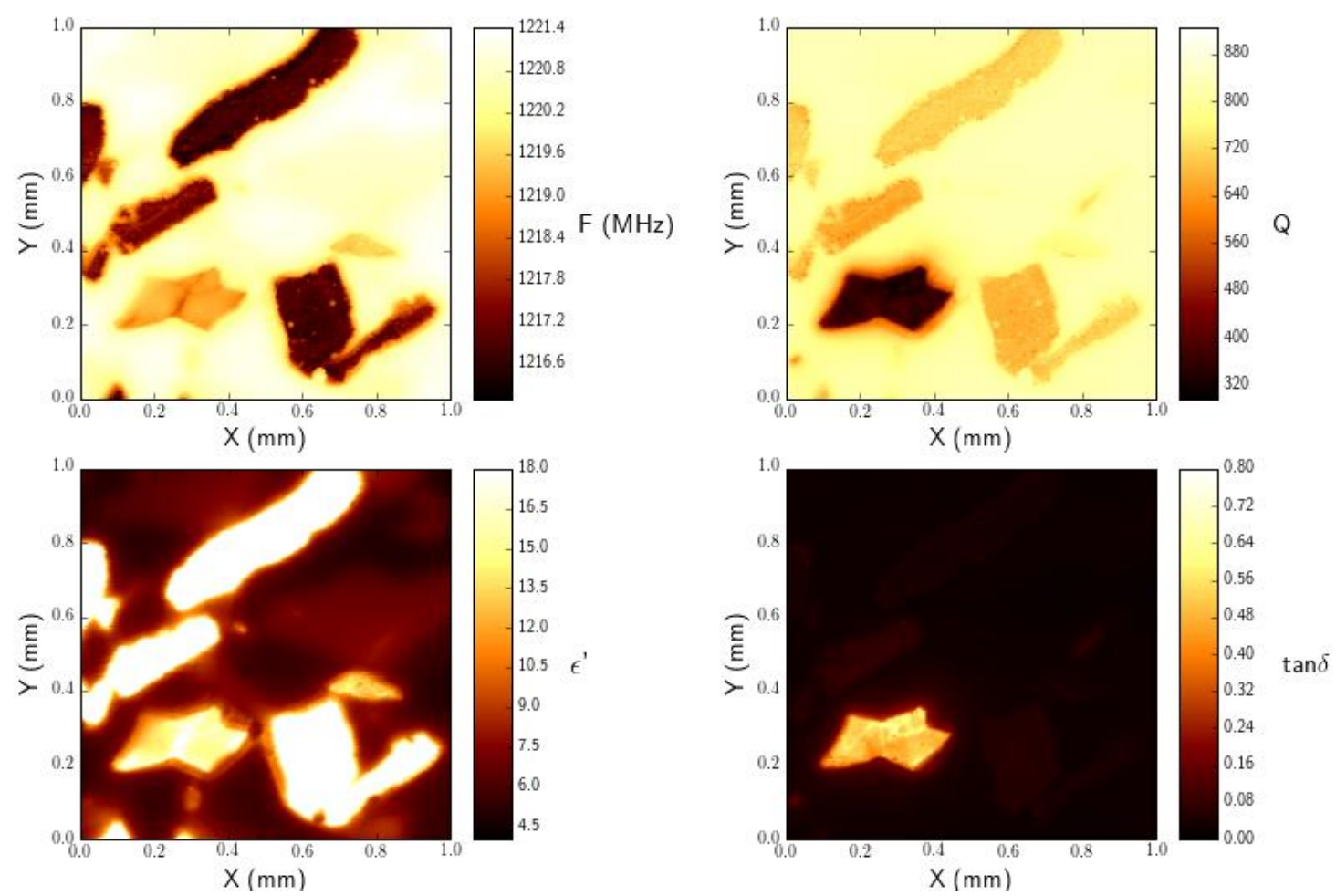

\section{A - Spherical tip}
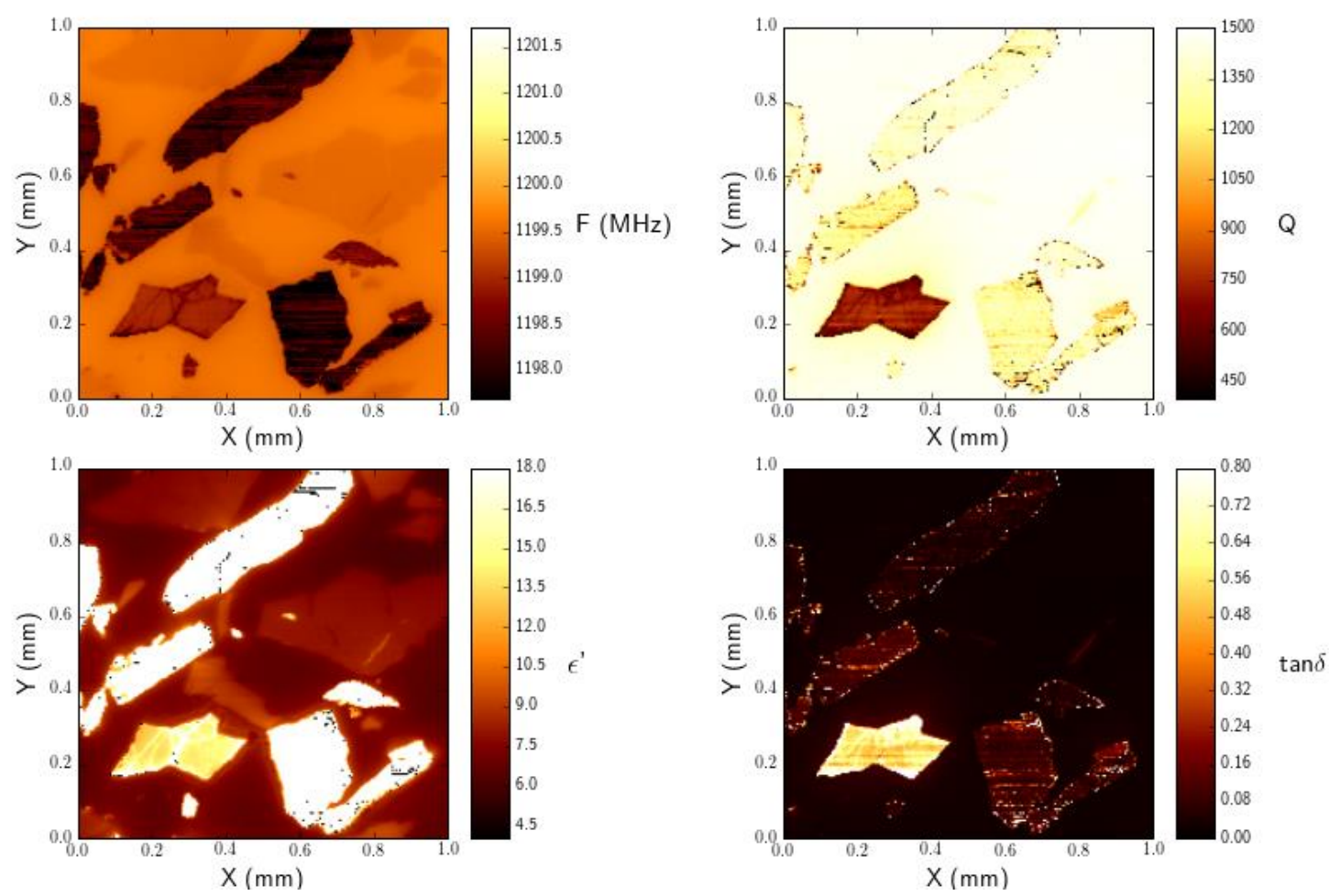

\section{B - Conical tip}

Fig. 12: 200x200 point image scans on a $1 \times 1 \mathrm{~mm}$ area of the rock specimen using the spherical and conical probe tips. Fig. 9 can be used to identify the mineral phases. Chalcopyrite can be seen to have particularly high loss. The data is clipped so points above and below the scale ranges are plotted in white and black respectively. Readings on magnetite areas are subject to large variations due to noise, especially for the conical tip (see text for further discussion). Calcite and apatite phases have low loss, and are not visible on the Q-factor and $\tan \delta$ plots using the scales used. 

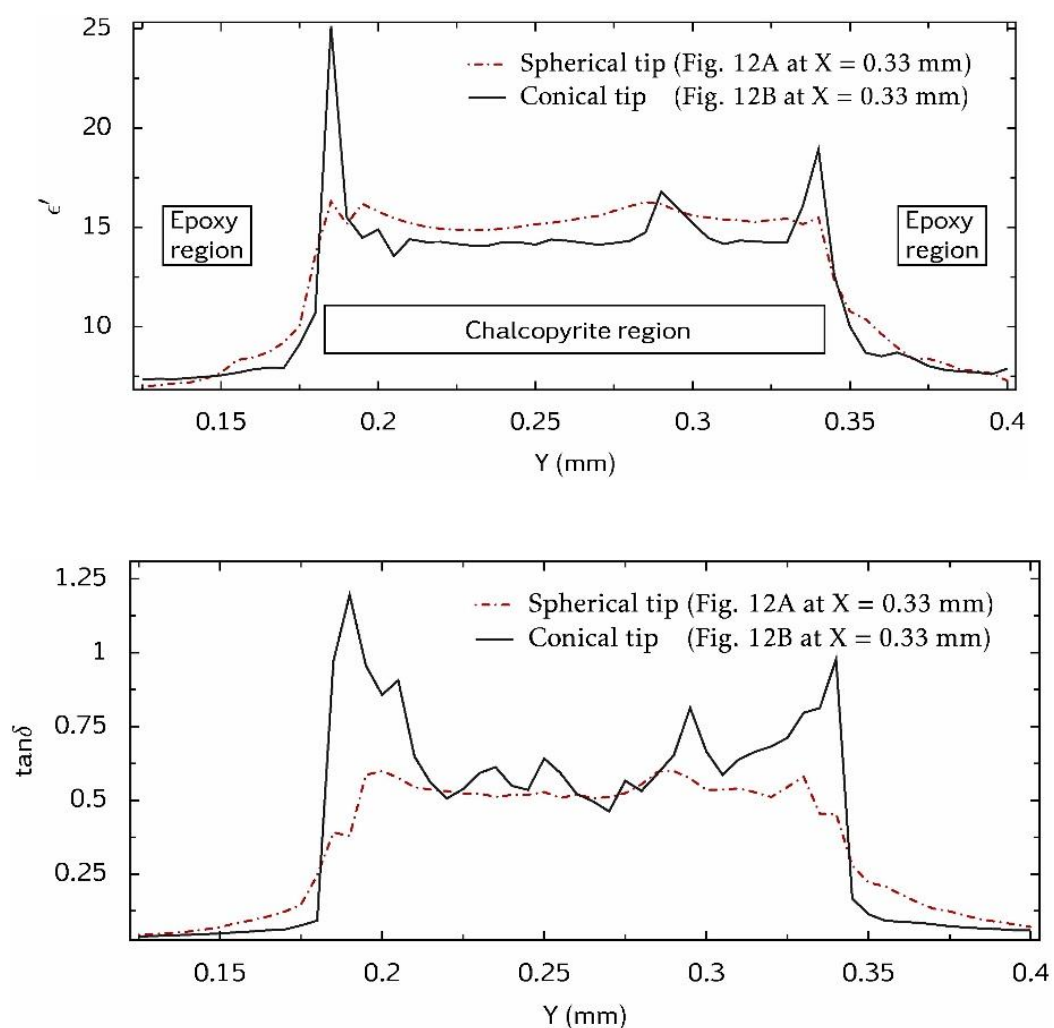

Fig 13: Linear scans across a chalcopyrite phase.
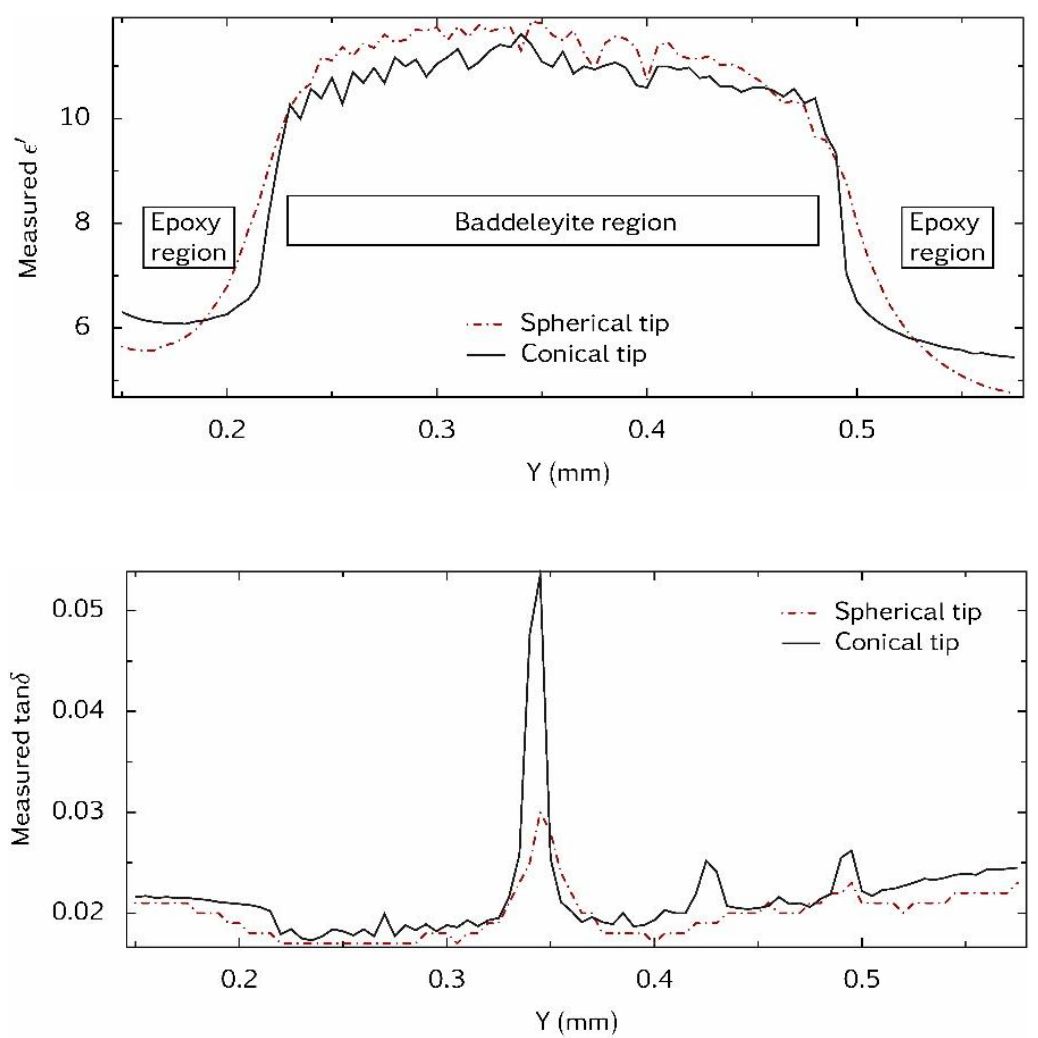

Fig 14: Linear scans across a baddeleyite phase. This phase has a vein running through it which has substantially elevated loss (which is shown with better resolution by the conical tip). 


\section{The effects of dielectric boundaries}

The approach curve and imaging data show local changes in permittivity can be measured with high resolution. However, calculations are based on a modelling assumption that the measured phases occupy an infinite half space. Observations show that it is generally sufficient for the thickness of specimens to be $\widetilde{\lessgtr} 10 x$ the tip diameter. For calibration reference specimens a slightly greater thickness (e.g. $\widetilde{\sim} 15 \mathrm{x}$ the tip diameter) ensures that the best calibration accuracy is obtained [11]. Thin specimens can sometimes be measured using the two-layer binary-tree model [37] if the complex permittivity of the substrate that supports them is known [11]. However, if a specimen is too thin in comparison to the diameter of the probe tip, the binary tree becomes so large that the calculation is unviable.

The effect of dielectric boundaries was evaluated by making scans on reference specimens from the centre of specimens until the tip reaches the edge (at which point the scanning is interrupted). The conical tip was used for these tests. The results (Fig. 15) show that, while the microscope has the ability to resolve details at a scale of $\approx 10 \mu \mathrm{m}$, a measurable reduction in apparent permittivity occurs when the distance is $\approx 1 \mathrm{~mm}$ from the boundary with air. The spatial range of this effect is comparable to the diameter of the hole in the top of the cavity and to the exposed length of the monopole probe (see Fig. 2). For lossy materials a similar boundary effect on $\tan \delta$ would be expected; however none of the reference specimens had sufficient loss to enable this to be observed. Effects on measurements caused by dielectric boundaries at these large distances are an inherent disadvantage of probing using a wire monopole - refer to Section I for more discussion. In the case of the rock specimen, the measured areas (Table 3 and Figs. 12-14) were at least $1 \mathrm{~mm}$ away from the edge of the specimen, so the proximity with the boundary with air should have minimal effect. The boundaries of the mineral phases within the rock sample must also have an effect, but this will be smaller because the permittivity of the epoxy matrix $\left(\varepsilon^{\prime}=2.94\right)$ is higher than that of air. Linear scans such as Figs. 13 and 14 show that there is negligible effect at distances $\widetilde{>} 0.1 \mathrm{~mm}$ inside the boundary with the surrounding epoxy matrix.

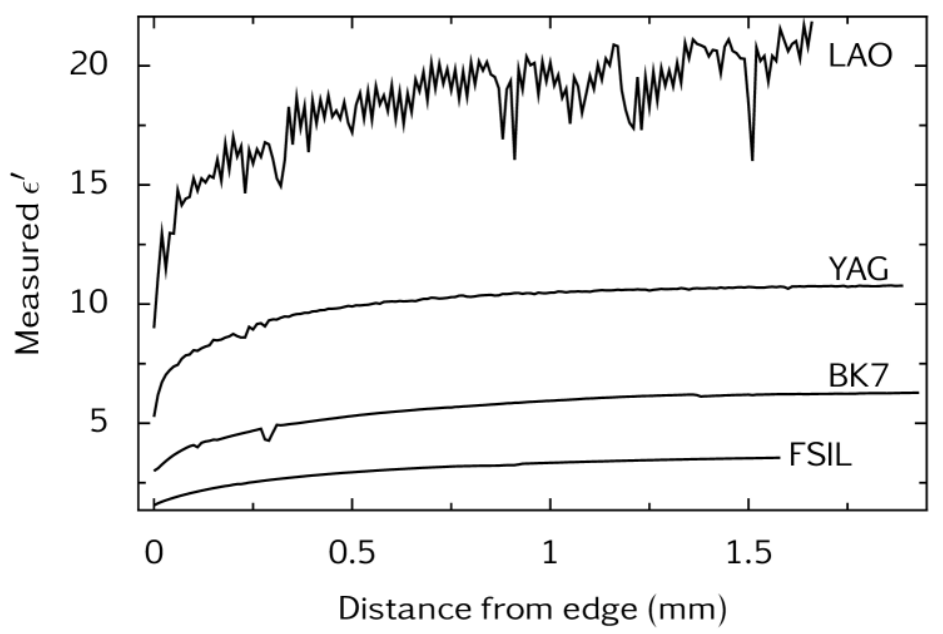

Fig. 15: Linear scans from the centre to the edge of the reference specimens. These were made using the conical tip. The curves show the effect of boundaries. Environmental vibrations have an increased effect for high permittivity specimens (Section III(d)) which is very evident for the LAO data.

\section{Conclusion}

Calibration and imaging experiments with a microwave microscope that has spherical and conical probe scanning probe tips were performed. The calibration model, which is based on an electrostatic model for a spherical tip, is found to be a less accurate fit for the conical tip; nevertheless accurate measurements of permittivity and loss tangent can be obtained. The conical tip geometry enables robust small diameter tips to be used, so the image resolution can be improved. Calibration and measurements were accomplished using a theoretical approach (based on complex frequency) that can be applied to the measurement of the permittivity and loss tangent of high loss materials. Scanned dielectric property images of the multiphase rock specimen were correlated with Mineral 
Liberation Analyser (MLA) results that identify the phases. Measurements on a multiphase material have been demonstrated on a scale of the order of $10 \mu \mathrm{m}$. The NSMM system described is best suited to measurements on materials with $\varepsilon^{\prime}<25$ and $0.005<\tan \delta<1$. It is unsuitable for measurements on conductive materials, such as magnetite and doped semiconductors. Uncertainties in $\varepsilon^{\prime}$ can be evaluated on the basis of measurements on reference specimens (Tables 1 and 2). For uniform solid specimens are large enough to avoid significant boundary effects, the uncertainty [38] in measured $\varepsilon^{\prime}$ for either tip is estimated to be $\pm 10 \%$ (at coverage factor $k=2$ ) for $\varepsilon^{\prime} \approx 10$. This corresponds to a confidence level of $95 \%$ assuming that results have a normal distribution. Solid lossy materials that are uniform on a small scale and so could be used as reference materials have not been identified, which makes it more difficult to evaluate the uncertainty of measurements of $\tan \delta$. In previous work [11] in which lossy polar liquids were measured, it was found that measurement errors $(\%)$ in $\tan \delta$ were typically twice as large as those in $\varepsilon^{\prime}$ (which in the authors' experience is typical for measurements by resonance). Loss tangent can be resolved to approximately 0.001 . Subject to this limit, the uncertainty of loss tangent measurements is therefore estimated to be typically $\pm 20 \%$ (at coverage factor $k=2$ ).

An apparent reduction in permittivity is observed at locations $\widetilde{\gtrless} 1 \mathrm{~mm}$ from the edges of uniform reference specimens. This is a result of the poor localisation of the E-fields between the monopole probe and the cavity lid. The size of the through-hole in the wall of the cavity $(1 \varnothing \mathrm{mm})$ and length of the probe outside the cavity were determined by practical considerations but clearly are limiting factors; if these dimensions were reduced the Efields would extend less far into space and the resolution would be improved. Similarly, measurements on one phase in a multiphase specimen are affected by penetration of E-fields into the surrounding (epoxy) matrix and other nearby phases. In general, phases $\widetilde{>} 0.2 \mathrm{~mm}$ across can be measured with minimal error provided that (i) the phase is located at least 1-mm away from the edge of the specimen and (ii) measurements on individual phases are made at least $0.1 \mathrm{~mm}$ inside the boundary with the epoxy matrix.

\section{Acknowledgements}

The NSMM was developed under the project "Electromagnetic Materials Measurements for Industrial Applications (EMINDA)". This was a 3-year project under the European Metrology Research Programme (EMRP) under Grant Agreement No. 217257. The EMRP is jointly funded by the EMRP participating countries within EURAMET and the European Union. Subsequent work and the preparation of this manuscript were funded by the Materials and Modelling programme of the UK Government's Department for Business, Energy and Industrial Strategy under contract NMS/MMS15.

The authors acknowledge assistance from a number of NPL colleagues; in particular J. W. Nunn and L. J. Brown for profile measurements on the probe tips using a confocal microscope.

\section{References}

[1] A. Imtiaz, T. M. Wallis and P. Kabos, "Near-field scanning microwave microscopy: An Emerging Research Tool for Nanoscale Metrology", IEEE Microw. Mag., vol. 15, no. 1, pp. 52-64, 2014, http://dx.doi.org/10.1109/MMM.2013.2288711

[2] C. Gao and X.-D. Xiang, "Quantitative microwave near-field microscopy of dielectric properties", Rev. Sci. Instrum., vol. 69, no. 11, pp. 3846-3851, 1998, http://dx.doi.org/10.1063/1.1149189

[3] A. K. Tagantsev, V. O. Sherman, K. F. Astafiev, J. Venkatesh and N. Setter, "Ferroelectric Materials for Microwave Tunable Applications", J. Electroceram., vol. 11, no. 1, pp. 5-66, 2003, http://dx.doi.org/10.1023/B:JECR.0000015661.81386.e6

[4] C. Gao, B. Hu, I. Takeuchi, K.-S. Chang, X.-D. Xiang, and G. Wang, "Quantitative scanning evanescent microwave microscopy and its applications in characterization of functional materials libraries", Meas. Sci. Tech., vol. 16, no. 1, pp. 248-260, 2005, http://dx.doi.org/10.1088/0957-0233/16/1/033

[5] J. Park, S. Hyun, A. Kim, T. Kim and K. Char, "Observation of biological samples using a scanning microwave microscope", Ultramicroscopy, vol. 102, no. 2, pp. 101-106, 2005, http://dx.doi.org/10.1016/j.ultramic.2004.09.007

[6] T. Monti, A. Tselev, O. Udoudo, I. N. Ivanov and S. W. Kingman, "High-resolution dielectric characterization of minerals: a step towards understanding the basic interactions between microwaves and rocks", International Journal of Mineral Processing, Vol 151, 2016, pp. 8-21, http://dx.doi.org/10.1016/j.minpro.2016.04.003

[7] S. W. Kingman and N. A. Rowson, "Microwave treatment of minerals-a review", Minerals Engineering, vol. 11, no. 11, pp. 1081-1087, 1998 http://dx.doi.org/10.1016/S0892-6875(98)00094-6

[8] J. Baker-Jarvis, M. D. Janezic, B. F. Riddle, R. T. Johnk, P. Kabos, C. L. Holloway, R. G. Geyer and C. A. Grosvenor, "Measuring the Permittivity and Permeability of Lossy Materials: Solids, Liquids, Metals, Building Materials and Negative Index Materials", NIST Technical Note 1536, 2004, http://dx.doi.org/10.6028/NIST.TN.1536

[9] J. B. Salsman, "Measurement of dielectric properties in the frequency range of $300 \mathrm{MHz}$ to $3 \mathrm{GHz}$ as a function of temperature and density", Proc. Symp. Microwave Theory Appl. Mater. Process., vol. 34, pp. 203-214, 1991 
[10] V. Schmidt, W. Wilting, M. Gruse and N. Wagner, "A cylindrical guarded capacitor for spectral permittivity measurements of hard rock samples in the MHz-range", Meas. Sci. Tech., vol. 26, no. 10, 2015, pp. 1390-1398, http://dx.doi.org/10.1088/0957-0233/26/10/105902

[11] A. P. Gregory, J. F. Blackburn, K. Lees, R. N. Clarke, T. E. Hodgetts, S. M. Hanham and N. Klein, "Measurement of the permittivity and loss of high-loss materials using a Near-Field Scanning Microwave Microscope", Ultramicroscopy, vol. 161, 2016, p137-145, http://dx.doi.org/10.1016/j.ultramic.2015.11.015

[12] D.-Y. Sheu, "Micro-spherical probes machining by EDM", J. Micromech. Microeng., vol. 15, no. 1, pp. 185-189, 2005, http://dx.doi.org/10.1088/0960-1317/15/1/026

[13] J. Kim, M. Kim, H. Kim, D. Song, K. Lee and B. Friedman, "Improving images from a near-field scanning microwave microscope using a hybrid probe", APL, vol. 83, pp. 1026-1028, 2003, http://dx.doi.org/10.1063/1.1595134

[14] G. Gramse, G. Gomila and L. Fumagalli, "Quantifying the dielectric constant of thick insulators by electrostatic force microscopy: effects of the microscopic parts of the probe", Nanotechnology, vol. 23, no. 20, 2012, article 205703, http://dx.doi.org/10.1088/0957-4484/23/20/205703

[15] C. Balusek, B. Friedman, D. Luna, B. Oetiker, A. Babajanyan, and K. Lee, "A three-dimensional finite element model of near-field scanning microwave microscopy", J. Appl. Phys., vol. 112, no. 8, article 084318, 2012 http://dx.doi.org/10.1063/1.4759253

[16] S. M. Hanham, A. Gregory, S. A. Maier, N. Klein, "A dielectric probe for near-field millimeter-wave imaging ", Infrared, Millimeter, and Terahertz Waves (IRMMW-THz), 37th International Conference, 2012, http://dx.doi.org/10.1109/IRMMW-THz.2012.6380378

[17] V. V. Talanov, A. Scherz, R. L. Moreland, Andrew R. Schwartz, "Noncontact dielectric constant metrology of low-k interconnect films using a near-field scanned microwave probe", Appl. Phys. Lett., vol. 88, article 192906, 2006, http://dx.doi.org/10.1063/1.2203238

[18] N. Klein, P. Lahl, U. Poppe, F. Kadlec, and P. Kužel , "A metal-dielectric antenna for terahertz near-field imaging", J. Appl. Phys., vol. 98, article 14910, 2005, http://dx.doi.org/10.1063/1.1978972

[19] R. Omarouayache, P. Payet, J. Raoult, and L. Chusseau , "Millimeter-wave near-field imaging with bow-tie antennas", Optics Express, vol. 23, no. 9, p12144-12151, 2015, http://dx.doi.org/10.1364/OE.23.012144

[20] J. Kim, M.-S. Kim, K. Lee, J. Lee, D. Cha and B. Friedman, "Development of a near-field scanning microwave microscope using a tunable resonance cavity for high resolution", Meas. Sci. Technol., vol. 14, 2003, p7-12

[21] D. E. Steinhauer, C. P. Vlahacos, F. C. Wellstood, S. M. Anlage, C. Canedy and R. Ramesh, "Quantitative imaging of dielectric permittivity and tunability with a near-field scanning microwave microscope", Rev. Sci. Instrum., vol. 71, no. 7, 2000, pp. 2751-2758, http://dx.doi.org/10.1063/1.1150687

[22] V. V. Talanov, A. Scherz, R. L. Moreland, and A. R. Schwartz, "A near-field scanned microwave probe for spatially localized electrical metrology", Appl. Phys. Lett, vol. 88, 2006, article 134106, http://dx.doi.org/10.1063/1.2189147

[23] J. Kim, K. Lee, B. Friedman and D. Cha, "Near-field scanning microwave microscope using a dielectric resonator", Appl. Phys. Lett., vol. 83, no. 5, 2003, pp. 1032-1034, http://dx.doi.org/10.1063/1.1597984

[24] A. P. Gregory, "Q-factor measurement using a vector network analyser", National Physical Laboratory (UK), Report MAT 58, 2016 (to be published)

[25] A. P. Gregory, J. F. Blackburn, K. Lees, R. N. Clarke, T. E. Hodgetts, S. M. Hanham and N. Klein, "A near-field scanning microwave microscope for measurement of the permittivity and loss of high-loss materials", $84^{\text {th }}$ Microwave Measurement Conference (ARFTG), 2014, http://dx.doi.org/10.1109/ARFTG.2014.7013419

[26] R. N. Clarke (Ed.), 2003, "A Guide to the characterisation of dielectric materials at RF and microwave Frequencies", The Institute of Measurement and Control (IMC) and The National Physical Laboratory (NPL), London, ISBN 0904457389.

[27] E. Hollmann, O. Vendik, A. Zaitsev and B. Melekh, "Substrates for high-Tc superconductor microwave integrated circuits", Supercond. Sci. and Technol., vol. 7, pp. 609-622, 1994, http://dx.doi.org/10.1088/0953-2048/7/9/001

[28] Q. Zhang, and P. J. McGinn, "Imaging of oxide dielectrics by near-field microwave microscopy", J. Eur. Cer. Soc., vol. 25, no. 4, 2005, pp. 407-416, http://dx.doi.org/10.1016/j.jeurceramsoc.2004.02.013

[29] J. Krupka, A. P. Gregory, O. C. Rochard, R. N. Clarke, B. Riddle and J. Baker-Jarvis, "Uncertainty of complex permittivity measure-ments by split-post dielectric resonator technique", J. Eur. Cer. Soc., vol. 21, no. 15, pp. 2673-2676, 2001, http://dx.doi.org/10.1016/S0955-2219(01)00343-0

[30] M. P. Jones, "Applied mineralogy - A quantitative approach", Graham and Trotman, London, 1987

[31] W. Petruk, "Applied mineralogy in the mining industry". Elsevier Science B.V., Amsterdam, New York, 2000

[32] W. Petruk, "Automatic image analysis for mineral beneficiation" J. Metals, vol. 40, pp. 29-31, 1988, http://dx.doi.org/10.1007/BF03259018

[33] W. Petruk, "The MP-SEM-IPS image analysis system", CANMET report 87-1E, CANMET, Dept Energy, Mines and Resources, Canada, 1986

[34] W. B. Westphal, "Dielectric constant and loss data", Report AFML-TR-74-250 Part III, Laboratory for Insulation Research, MIT, May 1977

[35] R. H. Church, W. E. Webb and J. B. Salsman, "Dielectric properties of low-loss materials", Report of Investigations 9194, Bureau of Mines, US Dept. of the Interior, 1988

[36] P. J. Harrop and J. N. Wanklyn, "The dielectric constant of zirconia", Brit. J. Appl. Phys., vol. 18, pp. 739-742, 1967

[37] C. Gao, B. Hu, P. Zhang, M. Huang, W. Liu and I. Takeuchi, "Quantitative microwave evanescent microscopy of dielectric thin films using a recursive image charge approach", Appl. Phys. Lett., vol. 84, no. 23, pp. 4647-4649, 2004, http://dx.doi.org/10.1063/1.1759389

[38] "The expression of uncertainty and confidence in measurement", United Kingdom Accreditation Service document M3003, $3^{\text {rd }}$ Ed., 2012 